\title{
Three-dimensional effects on extended states in disordered models of polymers
}

\author{
Francisco Domínguez-Adame \\ Departamento de Física de Materiales, Facultad de Físicas, Universidad Complutense, E-28040 Madrid, Spain \\ Enrique Diez and Angel Sánchez \\ Escuela Politécnica Superior, Universidad Carlos III de Madrid, C./ Butarque 15, E-28911 Leganés, Madrid, Spain
}

(Received 13 July 1994)

\begin{abstract}
We study electronic transport properties of disordered polymers in a quasi-one-dimensional model with fully three-dimensional interaction potentials. We consider such quasi-one-dimensional lattices in the presence of both uncorrelated and short-range correlated impurities. In our procedure, the actual physical potential acting upon the electrons is replaced by a set of nonlocal separable potentials, leading to a Schrödinger equation that is exactly solvable in the momentum representation. By choosing an appropriate potential with the same spectral structure as the physical one, we obtain a discrete set of algebraic equations that can be mapped onto a tight-binding-like equation. We then show that the reflection coefficient of a pair of impurities placed at neighboring sites (dimer defect) vanishes for a particular resonant energy. When there is a finite number of such defects randomly distributed over the whole lattice, we find that the transmission coefficient is almost unity for states close to the resonant energy, and that those states present a very large localization length. Multifractal analysis techniques applied to very long systems demonstrate that these states are truly extended in the thermodynamic limit. These results are obtained with parameters taken from actual physical systems such as polyacetylene, and thus reinforce the possibility of verifying experimentally theoretical predictions about the absence of localization in quasi-one-dimensional disordered systems.
\end{abstract}

\section{INTRODUCTION}

Transport properties of disordered systems have become a fascinating research topic since the generality of localization phenomena in one dimension (1D) was first questioned a few years ago. ${ }^{1-18}$ Opposite to the conventional view that in $1 \mathrm{D}$ random systems almost all eigenstates are exponentially localized (see, e.g., Ref. 19 and references therein), it is nowadays known that in disordered systems where disorder exhibits some kind of spatial correlation bands of extended states arise. Spatial correlation means that random variables are not independent within a given correlation length or, equivalently, that the noise is nonwhite. Furthermore, supression of localization by structural correlation has been found both in classical and quantum systems. In the quantum case, electronic transport has been of course the subject of most works. There exists at present much evidence that correlated disorder inhibits wave localization, and that bands of extended states appear in tightbinding Hamiltonians ${ }^{1-7,9-11}$ as well as in more elaborated multiband systems like those described by KronigPenney models. ${ }^{13,14}$ Similarly, the occurrence of superdiffusion $^{8}$ and reflectionless spin waves in Heisenberg chains ${ }^{15}$ has been recently reported. In the classical case, random harmonic chains also present a band of shortwave delocalized vibrations whenever correlated disorder occurs, ${ }^{12,16}$ giving rise to a strong enhancement of the thermal conductivity of the lattice. ${ }^{18}$ All these theoretical analyses clearly demonstrate that transport properties in random systems where structural correlations are present are very different to what is found in ordinary random systems. It is also clear that supression of localization does not depend on the classical or quantum nature of the system and therefore structural correlations are to be regarded as the origin of this unexpected feature.

In spite of the already available body of theoretical work, the physical relevance of these extended states is still unknown. To our knowledge, there is no experimental evidence whatsoever of the existence of these states and their influence in measurable transport properties. We regard this as the key question to be posed about the theoretical results, above the more fundamental one on the nature of these states in infinite systems. Notwithstanding, we have addressed both issues in our research project on disordered systems ${ }^{12-14,17,18,20-22}$ and in particular in the work we are reporting here. Regarding experimental demonstration of delocalization, we have recently shown how the bands of extended states must reveal themselves through characteristic features in the dc conductance of disordered superlattices at finite temperature. ${ }^{17}$ Moreover, we have also found that short-range correlated disorder has profound effects on coherent $^{20}$ and incoherent ${ }^{22}$ trapping, as well as on the optical properties ${ }^{21}$ of excitons. In the same spirit, we have even proposed mechanical analogs where classical extended vibrations should be found. ${ }^{18} \mathrm{We}$ note that, aside from the basic research goal of finding out whether delocalization actually occurs in real physical systems, we also have in mind an applied aim, namely, verifying 
whether correlated disorder gives rise to particular features that can be used for new devices or applications.

In this work, we report further progress along the lines in the preceding paragraph. Searching for physically realizable systems where delocalization may play a crucial role, we turn ourselves to one of the pioneering works in the field, namely, the work of Phillips and $\mathrm{Wu}$ on polyaniline (see Ref. 5 and references therein). These authors showed that polyaniline could be mapped onto a tight-binding, random dimer model that has a band of extended states, originated by a resonance at a single dimer defect. It is evident that, on the one hand, similar mappings can be worked out for different polymers and, on the other hand, that delocalized bands have to affect their conductance properties. Indeed, Wu and Phillips argued that the fact that polyaniline was a conducting polymer was closely related to this unexpected delocalization phenomena. ${ }^{4}$ Their calculations were carried out in the framework of a purely 1D tight-binding Hamiltonian. However, although it is quite reasonable to approximate the structure of a polymer by a line, it is also true that the physics involved is three dimensional (3D) and that actually the linear structure of polymers is not straight but folds and wanders in $3 \mathrm{D}$ space. It is then natural to ask whether the above theoretical results will still hold when more realistic models including $3 \mathrm{D}$ effects are considered. The answer to this question is very important if $\mathrm{Wu}$ and Phillips's results are to be compared to measurements on real polymers: If the delocalized band is destroyed by $3 \mathrm{D}$ effects, then their theoretical results are merely academic, and worse, any possible technological application becomes very unlikely. On the other hand, this question is not without interest from the fundamental viewpoint. As we mentioned above, localization of almost all eigenstates by uncorrelated disorder is expected in 1D random systems, but three-dimensional (3D) systems require a minimum amount of disorder to give rise to localization. ${ }^{19}$ Then a question arises in a natural way within this context, namely, the possible effects of correlated disorder on 3D eigenstates. As far as we know, this problem has been already studied by Stephens and Skinner, ${ }^{23}$ who found that tight-binding Hamiltonians with short-range correlated diagonal disorder in a cubic lattice presents a localization threshold that is independent of the amount of correlation. This finding seems to indicate that the influence of structural correlations is relevant only to pure $1 \mathrm{D}$ systems, which adds further interest to the elucidation of the applicability of Wu and Phillips's results.

We address the above issues by introducing a completely general model to study electronic properties in random systems based on the so-called nonlocal (separable) potential (NLP) method, in which the actual potential at each site of an arbitrary lattice is replaced by a projective operator. ${ }^{24-26}$ The treatment is fully $3 \mathrm{D}$, although we restrict ourselves to a linear chain, and so our model is not a 1D model in the traditional sense as we have here an array of $3 D$ potentials along a straight line. Moreover, the model can be straightforwardly extended to folded (i.e., nonstraight) systems by appropriate choices of the parameters. As a major point, we will demonstrate the occurrence of a well-defined band of extended states in the electronic energy spectrum due to structural correlations, in spite of the $3 \mathrm{D}$ character of the equation of motion. In particular, we consider the case in which pairs of impurities (the so-called dimer defects) are placed at random in an otherwise perfect lattice. The location of the band of the extended states in the electronic spectrum is determined from the condition of vanishing of the reflection coefficient from a single dimer defect.

We note that our model is monoelectronic, and therefore it is not suitable to include the different nonlinear excitations present in polymers in general, like solitons, polarons, and bipolarons. ${ }^{27}$ If one is interested in the effect of these types of excitations, it would be necessary to resort to other models like, e.g., many-body Hamiltonians like the Su-Schrieffer-Heeger (SSH) or the PeierlsHubbard ones (see Ref. 27 and references therein). In this context, it is important to clarify that electron-electron correlations are not needed to generate solitons, at least charged solitons: Thus, for instance, when a single electron is added to the SSH model, a polaron forms, whereas two electrons are required (although they may be noninteracting) to form a soliton. We also notice that our treatment involves neglecting all thermal degrees of freedom (electron-phonon coupling and local lattice distortions) which is enough for the purposes of the present paper. On the other hand, what we are interested in is delocalization effects due to the presence of paired impurities, and in showing them as clean and isolated as possible. With that goal in mind, it is very reasonable to focus on linear excitations (those described by our model, as well as the model by $\mathrm{Wu}$ and Phillips), as it is well known that nonlinear waves are much more robust in the presence of disorder. ${ }^{28}$ This is even more so for kinklike solitons, which are the ones found in polymers, whose topological charge prevents their anihilation and reduces the effects of disorder to, possibly, pinning (see, for instance, Ref. 29 and references therein). We believe that localization will affect first linear excitations and thus we study the model we describe below. The fact that we use parameters for polyacetylene later is because we have obtained them in the framework of our nonlocal potential model with great accuracy, ${ }^{26}$ and therefore they are already available to present an example of the orders of magnitude to be expected in polymer applications; however, our calculations should apply qualitatively to many other polymer systems.

The paper is organized as follows. In Sec. II we present our model and summarize previous work ${ }^{26}$ that is necessary for a better understanding of the present paper. After describing how nonlocal potentials can be used to model 3D systems, we consider the scattering from a single dimer defect exactly and find the resonance condition for perfect transparency. We close this section with a brief account of exact expressions to compute the physical magnitudes of interest in a lattice containing a certain number of dimer defects. Afterwards, in Sec. III we concern ourselves with our main topic, the random quasione-dimensional lattice with paired disorder. We present our numerical results demonstrating the existence of extended states via transmission and Lyapunov coefficients, 
which relate to physically relevant quantities such as localization length, as well as multifractal analysis, which points out the character of these states in the infinitesize limit: Thus, we show how the bands of extended states reveal themselves through well-defined peaks in the transmission coefficient versus energy plots, and how the truly extended character of those states is also demonstrated in their scaling properties. Section IV concludes the paper with a brief summary of the main results and some possible applications in different physical contexts.

\section{NLP APPROACH} TO MULTICENTER INTERACTIONS

The starting point for the NLP procedure is the Schrödinger equation for multicenter potentials, corresponding to the physical situation we want to gain insight into. The solution of this kind of problem is of widespread interest not only in condensed matter physics but also in atomic or molecular physics. As is well known, such a solution is expected to involve enormous intricacies since in most cases prohibitively cumbersome calculations are needed. Several methods have been developed to study the motion of electrons in a given superposition of $3 \mathrm{D}$ potentials. Among them, the NLP approach is the natural generalization of the famous Kronig-Penney model ${ }^{30}$ to the $3 \mathrm{D}$ case. This method leads to an exactly solvable Schrödinger equation from which the electron energy can be obtained in a closed form. What is more important, it is always possible to find a NLP (or a sum of them) which reproduces any set of given electronic states, ${ }^{31}$ and so there is no theoretical limit to the numerical accuracy with which physical results may be obtained. We first summarize the NLP formalism and then discuss its application to conducting polymers.

\section{A. Schrödinger equation for the NLP}

We begin with the Schrödinger equation for the NLP, which reads as follows ${ }^{26}$ (we take $\hbar=m=1$ hereafter):

$$
\begin{aligned}
\left(\mathbf{p}^{2}-2 E\right) \psi(\mathbf{r})= & \sum_{k} \lambda_{k} V\left(\left|\mathbf{r}-\mathbf{R}_{k}\right|\right) \\
& \times \int d^{3} r^{\prime} V\left(\left|\mathbf{r}^{\prime}-\mathbf{R}_{k}\right|\right) \psi\left(\mathbf{r}^{\prime}\right),
\end{aligned}
$$

where $\mathbf{R}_{k}$ denotes the position of each lattice site and $\lambda_{k}$ is the corresponding coupling constant. We will immediately see how Eq. (1) connects with the physical problem of interest through suitable choices of the potential $V$. For simplicity we have assumed that the function $V$ is spherically symmetric, although more complicated symmetries can be also easily handled. In Fourier space we have

$$
\psi(\mathbf{p})=\left(\frac{1}{\mathbf{p}^{2}-2 E}\right) \sum_{k} \lambda_{k} V(p) \exp \left(-i \mathbf{p} \cdot \mathbf{R}_{k}\right) \chi_{k},
$$

where

$$
\chi_{k}=\int d^{3} p V^{*}(p) \exp \left(i \mathbf{p} \cdot \mathbf{R}_{k}\right) \psi(\mathbf{p})
$$

Here $\psi(\mathbf{p})$ and $V(p)$ denote the Fourier transforms of $\psi$ and $V$, respectively. The asterisk means complex conjugation; the Fourier transform of real and spherically symmetric functions is also real, but we retain it should nonspherical functions be considered. The coefficients $\chi_{k}$ are the quantities of interest, since we will show that they are related to the wave function in real space. We will be more specific about their meaning after we have specified the potential $V(p)$ and computed the corresponding equations for $\chi_{k}$. Inserting Eq. (2) in Eq. (3) we obtain the following set of algebraic equations for the parameters $\chi_{k}$ :

$$
\chi_{k}=\sum_{j} \lambda_{j} \int d^{3} p \frac{|V(p)|^{2}}{\mathbf{p}^{2}-2 E} \exp \left[i \mathbf{p} \cdot\left(\mathbf{R}_{k}-\mathbf{R}_{j}\right)\right] \chi_{j}
$$

Due to the spherical symmetry of the potential, the angular integration can be carried out in Eq. (4). In so doing, we finally obtain

$$
\chi_{k}=\sum_{j} 4 \pi \lambda_{j} \int_{0}^{\infty} d p \frac{p^{2}|V(p)|^{2}}{\mathbf{p}^{2}-2 E} \frac{\sin p R_{k j}}{p R_{k j}} \chi_{j}
$$

where $R_{k j} \equiv\left|\mathbf{R}_{k}-\mathbf{R}_{j}\right|$. It is understood that the factor $\left(\sin p R_{k j}\right) / p R_{k j}$ is replaced by 1 when $k=j$, that is, by its limit as $R_{k j} \rightarrow 0$.

\section{B. Application to quasi-one-dimensional polymer models}

At this point we should stress that Eq. (5) is completely general, once the potential $V(p)$ is specified. For a given $1 \mathrm{D}, 2 \mathrm{D}$, or $3 \mathrm{D}$ lattice $\left\{\mathbf{R}_{k}, k=1,2, \ldots, N\right\}, N$ being the number of sites, the eigenenergies can be found by solving the secular equation arising from the $N \times N$ symmetric determinant associated with Eq. (5). In this fashion, we arrive at the key of the NLP procedure: The crucial question is to set up an appropriate potential $V(p)$ that reproduces the observed energy values of the physical situation being considered. For instance, we have previously found that the Yamaguchi's NLP (Ref. 32) is most appropriate to describe Coulomb bound states (see Ref. 26), whereas surface $\delta$-function potentials, that is, a force field vanishing everywhere except on a spherical shell of radius $R$, are very well suited to simulate electron potentials on long quasi-one-dimensional polymers, as polyacetylene or polyaniline. ${ }^{26}$ This is the case we are interested in, and therefore we concentrate ourselves on this choice of potential from now on; i.e., we take

$$
V(r)=\frac{1}{r^{2}} \delta(r-R), \quad V(p)=\sqrt{\frac{2}{\pi}} \frac{\sin p R}{p R} .
$$

Plugging this potential into Eq. (5) we get

$$
\chi_{k}=8 \frac{\lambda_{k}}{R^{2}} A(E) \chi_{k}+\sum_{j \neq k} 8 \frac{\lambda_{j}}{R^{2}} B_{k j}(E) \chi_{j},
$$


where for brevity we have defined

$$
\begin{aligned}
A(E) & =\int_{0}^{\infty} d p \frac{\sin ^{2} p R}{\mathbf{p}^{2}-2 E}=\frac{\pi}{4 \kappa}\left(1-e^{-2 \kappa R}\right), \\
B_{k j}(E) & =\int_{0}^{\infty} d p \frac{\sin ^{2} p R}{\mathbf{p}^{2}-2 E} \frac{\sin p R_{k j}}{p R_{k j}} \\
& =\frac{\pi \exp \left(-\kappa R_{k j}\right)}{4 \kappa^{2} R_{k j}}(\cosh 2 \kappa R-1) .
\end{aligned}
$$

We are restricting ourselves to the case of interest, namely, $E<0$, and then $\kappa \equiv \sqrt{-2 E}$ is a real parameter.

We note that interference effects due to the interaction of the electron with the lattice appear in the coefficients $B_{k j}(E)$ : The larger the distance between site $k$ and $j$, the smaller the corresponding coefficient. In other words, such coefficients are rapidly decreasing functions of $R_{k j}$ whenever $\kappa$ is not very small (deep potentials). In our problem, this is a good approximation, and hence, to simplify numerical analysis, we assume that only nearestneighbor interactions along the linear lattice are significant and write

$$
\begin{aligned}
\chi_{k}= & 8 \frac{\lambda_{k}}{R^{2}} A(E) \chi_{k}+8 \frac{\lambda_{k+1}}{R^{2}} B_{k k+1}(E) \chi_{k+1} \\
& +8 \frac{\lambda_{k-1}}{R^{2}} B_{k k-1}(E) \chi_{k-1} .
\end{aligned}
$$

To evaluate the coupling constant $\lambda_{k}$ in terms of experimentally measurable quantities, we consider an isolated potential centered at $\mathbf{R}_{k}$. This we accomplish by neglecting the interaction with other lattices sites, and so we take $B_{k \neq 1}(E) \rightarrow 0$ in Eq. (9), thus obtaining the condition determining the energy of bound states $E_{k}$, namely, $8 \lambda_{k} A\left(E_{k}\right) / R^{2}=1$. This is a transcendental equation which can be easily solved numerically, but it leads to a simpler expression for small values of $R$, a limiting case we will consider later. For a small radius $R$ we can expand $A\left(E_{k}\right)$ to obtain a relationship between the coupling constant $\lambda_{k}$ and the energy of the (single) bound state $E_{k}=-\kappa_{k}^{2} / 2$ :

$$
\lambda_{k} \sim \frac{R}{4 \pi}\left(1+\kappa_{k} R\right) .
$$

Finally, inserting Eq. (10) in Eq. (9) and taking the limit $R \rightarrow 0$ in such a way that $\kappa_{k}$ remains constant, we obtain the following tight-binding-like equation for the coefficients $\chi_{k}$ :

$$
\begin{aligned}
\left(\kappa-\kappa_{k}\right) \chi_{k}= & \frac{\exp \left(-\kappa R_{k k+1}\right)}{R_{k k+1}} \chi_{k+1} \\
& +\frac{\exp \left(-\kappa R_{k k-1}\right)}{R_{k k-1}} \chi_{k-1} .
\end{aligned}
$$

Note that in this tight-binding set of equations the transfer integrals depend exponentially on the distance between nearest-neighbor distances, as should be expected. This is consistent with our previous disregard of longerrange interactions. We can use these equations to describe the dynamics of electrons in the presence of diago- nal as well as off-diagonal randomness. In the rest of the paper, and without loss of validity, we further assume diagonal disorder, which implies that $R_{k \neq 1}=L, L$ being the lattice parameter. Defining $\rho \equiv \kappa L$ and $\rho_{k} \equiv \kappa_{k} L$ for the sake of brevity, we thus arrive at

$$
\left(\rho-\rho_{k}\right) e^{\rho} \chi_{k}=\chi_{k k+1}+\chi_{k k-1} .
$$

It is most important to stress that the number of free parameters appearing in these equations of motion has been kept to a minimum: We have only introduced the strength of the potential (which manifests itself in the value of the single bound state level, appearing in $\rho_{k}$ ) and the lattice parameter $L$.

Before we proceed to study the above equations of motion for our $3 \mathrm{D}$ model polymer, we now clarify the physical meaning of the coefficients $\chi_{k}$. From their definition in Eq. (3) and the Parseval identity ${ }^{33}$ we have

$$
\chi_{k}=\int d^{3} r V(r) \psi\left(\mathbf{r}+\mathbf{R}_{k}\right)
$$

with $V(r)=\left(1 / r^{2}\right) \delta(r-R)$. In the limit $R \rightarrow 0$ one gets $V(r) \rightarrow\left(1 / r^{2}\right) \delta(r)=\delta(r)$. Therefore, in that limiting case

$$
\chi_{k}=\psi\left(\mathbf{R}_{k}\right)
$$

We thus see that $\chi_{k}$ is nothing but the value of the electron wave function at site $\mathbf{R}_{k}$, which is of course the quantity of interest. On the other hand, this is the reason of the denomination of Eq. (12) as tight-binding equations of motion.

\section{Perfect quasi-one-dimensional lattices}

Before considering random lattices, it is instructive to study the case of perfect lattices, that is, those lattices with $\rho_{k}=\rho_{0}$. Since there exists translational symmetry, the Bloch theorem holds and we look for solutions of the form $\chi_{k}=U \exp (i Q L k), Q$ being the crystal momentum and $U$ a constant. Inserting this solution in Eq. (14) we readily obtain the dispersion relation

$$
\cos Q L=\frac{e^{\rho}}{2}\left(\rho-\rho_{0}\right) .
$$

Real values of $Q$, obtained by usual numerical methods give us the electron energy $E=-\rho^{2} / 2 L^{2}$ as a function of $Q$ and, consequently, the band structure of the lattice. To check the validity of the tight-binding approach we have assumed, it is necessary to compare this band structure with that obtained by including all non-nearest-neighbor interactions. We have already calculated it in Ref. 26, obtaining

$$
\cos Q L=\cosh \rho-\frac{1}{2} e^{\rho_{0}}
$$

Assuming that the lattice parameter $L$ is large and the potentials are deep (the basic assumptions in the tightbinding approach) it becomes clear that $\rho_{0}$ and $\rho$ are 
large but the difference $\rho-\rho_{0}$ is small. With this assumptions it is a matter of simple algebra to demonstrate that Eq. (16) reduces to Eq. (15). This leads us to the conclusion that one can confidently use Eq. (15) to describe the motion of tightly bound electrons in a lattice.

From an experimental point of view, comparison with measurements of real polymers requires the evaluation of the two input parameters, namely, $L$ and $\rho_{0}$, from experimental data. The first one is usually known from $\mathrm{x}$-ray data and, in principle, it is easy to obtain. The second one requires more information on the electronic band structure obtained, for instance, from spectroscopy measurements. Let us assume for the moment that the energies of the experimental band edges are known, and let $E_{t}$ and $E_{b}$ be the energy of the top and the bottom of the band in the perfect lattice, respectively $(Q L=\pi$ and $Q L=0)$, and $\rho_{t}=L \sqrt{-2 E_{t}}$ and $\rho_{b}=L \sqrt{-2 E_{b}}$. Using Eq. (15) one has the relationship

$$
\rho_{0}=\frac{\rho_{t} \exp \left(\rho_{t}\right)+\rho_{b} \exp \left(\rho_{b}\right)}{\exp \left(\rho_{t}\right)+\exp \left(\rho_{b}\right)}
$$

Therefore, from knowledge of the experimental band edges we can calculate the semiempirical parameter $\rho_{0}$. We have thus shown how the model parameters can be found for comparison to the particular polymer one is interested in. We will make use of this result later.

\section{Scattering from a single dimer defect}

As mentioned in the Introduction, we are interested in the effects of structural correlations on the localization properties of quasi-one-dimensional polymer models. Following Wu and Phillips, ${ }^{4}$ the simplest way to consider structural correlations is to introduce impurities at random but in pairs of sites. Physically this would correspond, for instance, to complexes of defects frequently encountered not only in polymers but also in molecular and solid state physics. In particular, a very clear description of dimer defects in polyaniline can be found in Ref. 5. In our model this means that $\rho_{k}$ can take only two values, $\rho_{0}$ and $\rho_{0}^{\prime}$, with the additional constraint that $\rho_{0}^{\prime}$ appears only in pairs of neighboring sites, which we will refer to as dimer defects.

Let us consider a single dimer defect placed at sites $k=0$ and $k=1$ in an otherwise perfect lattice. To proceed, we have to take into account in the first place the condition for an electron to move in the perfect lattice which, recalling Eq. (15), is given by

$$
\left|\frac{e^{\rho}}{2}\left(\rho-\rho_{0}\right)\right| \leq 1
$$

this constraint gives the allowed energy values once $\rho_{0}$ is fixed. Now considering the equation of motion, Eq. (12), at $k=-1,0,1$ and eliminating $\chi_{0}$ and $\chi_{1}$ one gets

$$
-\chi_{2}=\left(\Omega+\Omega^{\prime}-\Omega \Omega^{\prime 2}\right) \chi_{-1}-\left(1-\Omega^{\prime 2}\right) \chi_{-2},
$$

where we have defined $\Omega \equiv e^{\rho}\left(\rho-\rho_{0}\right)$ and $\Omega^{\prime} \equiv e^{\rho}\left(\rho-\rho_{0}^{\prime}\right)$ for brevity. Besides a constant phase factor of $\pi$, Eq. (19) reduces to the equation of motion in the perfect lattice whenever $\Omega^{\prime}=0$, in which sites $k=0$ and $k=1$ have been eliminated. This means that the reflection coefficient at the single dimer vanishes, and consequently there exists a complete transparency. This occurs only for a particular energy of the incoming electron, $E_{r} \equiv$ $-\rho_{r}^{2} / 2 L^{2}$, given by the condition $\Omega^{\prime}=0$, i.e., $\rho_{r}=\rho_{0}^{\prime}$. Hence this resonance effect occurs whenever the incoming electron matches the energy level of the (isolated) impurity, and this is possible only if $\left|\rho_{0}-\rho_{0}^{\prime}\right| \leq 2 \exp \left(-\rho_{0}^{\prime}\right)$, as seen from Eq. (18). This is to be compared with both the results of Ref. 4, where a single resonant energy is found as well, and to those in Refs. 13 and 14 for a continuum Kronig-Penney random dimer model, where an infinite number of resonances arise. We see that delocalization effects of structural correlation may be more or less dramatic depending on the physical situation studied. On the other hand, the important result is that the resonance of the simple $1 \mathrm{D}$ tight-binding random dimer model is preserved in our $3 \mathrm{D}$ setup.

\section{E. Scattering from a lattice with random dimer defects}

We now proceed to the problem of a random lattice with a finite number of dimer defects. Of course, the above results do not imply anything about extended states in a lattice with a finite number of dimers defects, and it is necessary to study that problem separately. For definiteness, we introduce the concentration of defects, $c$, given by the ratio between the total number of impurities (twice the number of dimer defects) and the total number of sites, $N$, in the lattice. We introduce this definition to facilitate direct comparison with results in ordinary random lattices with the same number of impurities and thus the same value of $c$, although in the latter case there are no constraints on the random location of the impurities. To study the transmission properties of electrons through the random lattice, we place it between two semi-infinite perfect lattices. Therefore we introduce the reflection $r$ and transmission $t$ amplitudes through the relationships

$$
\chi_{k}= \begin{cases}e^{i \rho k}+r e^{-i \rho k}, & k<1 \\ t e^{i \rho k}, & k>N .\end{cases}
$$

To determine both amplitudes we use the well-known transfer-matrix techniques (see, e.g., Ref. 34). Thus we cast Eq. (12) into the matrix form

$$
\left(\begin{array}{c}
\chi_{k+1} \\
\chi_{k}
\end{array}\right)=\left(\begin{array}{cc}
\alpha_{k} & -1 \\
0 & 1
\end{array}\right)\left(\begin{array}{c}
\chi_{k} \\
\chi_{k-1}
\end{array}\right) \equiv P_{k}\left(\begin{array}{c}
\chi_{k} \\
\chi_{k-1}
\end{array}\right)
$$

where $\alpha_{k}=\left(\rho-\rho_{k}\right) e^{\rho}$. The transfer matrix of the whole lattice is then found as

$$
T(N)=\prod_{k=N}^{1} P_{k}
$$

which relates the wave function at both edges of the lat- 
tice. Using the fact that $\operatorname{det}(T)=1$ we finally arrive at the following expression for the transmission coefficient $\tau=|t|^{2}$ :

$$
\tau=\tau(E)=\frac{4 \sin ^{2} \rho}{D(E)}
$$

with

$$
\begin{aligned}
D(E)= & T_{11}^{2}+T_{12}^{2}+T_{21}^{2}+T_{22}^{2} \\
& +2\left(T_{11} T_{12}+T_{21} T_{22}-T_{11} T_{21}-T_{12} T_{22}\right) \cos \rho \\
& -2\left(T_{11} T_{22}+T_{12} T_{21}\right) \cos ^{2} \rho+2 \sin ^{2} \rho,
\end{aligned}
$$

where we have dropped the explicit dependence on $N$ of the transfer-matrix elements. The transmission coefficient $\tau$ can be recurssively computed from the matrix elements of $T(N)$; taking into account the fact that $T(N)=P_{N} T(N-1)$ and $T(0)=P_{0}$ we find the following recurrence relations involving only real parameters:

$$
\begin{aligned}
& T_{11}(N)=\alpha_{N} T_{11}(N-1)-T_{11}(N-2), \\
& T_{12}(N)=\alpha_{N} T_{12}(N-1)-T_{12}(N-2), \\
& T_{21}(N)=T_{11}(N-1), \\
& T_{22}(N)=T_{12}(N-1), \quad N=2,3, \ldots,
\end{aligned}
$$

with the initial conditions $T_{i j}(0)=\delta_{i j}, T_{11}(1)=\alpha_{1}$, $T_{12}(1)=-1, T_{21}(1)=1$, and $T_{22}(1)=0$.

Other physically relevant magnitudes can be readily obtained from the transfer matrix $T(N)$. In particular the Lyapunov coefficient, which represents the rate of the growth of the wave function, is nothing but the inverse of the localization length. It can be computed as (measured in units of $L^{-1}$ ) (Ref. 35)

$$
\Gamma(E)=\left(\frac{1}{N}\right)\left(T_{11}^{2}+T_{12}^{2}+T_{21}^{2}+T_{22}^{2}\right) .
$$

Delocalization of the electronic wave function is seen through the decrease of this parameter.

The results we have obtained so far provide an exact, although nonclosed, analytical description of any random lattice with correlated as well as uncorrelated disorder. With them, we can compute the magnitudes we mentioned above. All expressions are very simple and suitable for an efficient numerical treatment for any specific case. We will now evaluate them for several interesting cases to describe the relevant features of the model and indications of extended states.

\section{RESULTS AND DISCUSSIONS}

There are five parameters that can be varied in our model, namely, the lattice constant $L$, the strengths of the scatterers, $\rho_{0}$ and $\rho_{0}^{\prime}$, the total number of scatterers, $N$, and the defect concentration $c$. In order to find results as close as possible to actual systems, we consider a quasi-one-dimensional polymer, as is the case of polyacetylene $(\mathrm{CH})_{x}$, which has been the focus of most of the experimental and theoretical works. ${ }^{36}$ In a perfect lattice, taking a uniform carbon-carbon bond length of $L=1.39 \AA,{ }^{37}$ we have previously estimated ${ }^{26}$ that $\rho_{0}=1.466$, corresponding to an energy level of the isolated potential of $-4.23 \mathrm{eV}$. We can confidently take these values as correct since the predicted effective mass is found to be $m^{*}=1.65$, in excellent agreement with the experimental result $m^{*}=1.7 \pm 0.1$. As an example, we will consider $\rho_{0}^{\prime}=1.550$, implying an energy level of the isolated impurity of $-4.73 \mathrm{eV}$. Note that the condition $\left|\rho_{0}-\rho_{0}^{\prime}\right| \leq 2 \exp \left(-\rho_{0}^{\prime}\right)$ holds; that is, the energy level of the isolated impurity lies in the band of the perfect lattice. Hence, according to our previous considerations, there exits complete transparency at an incoming energy of $-4.73 \mathrm{eV}$ if only a single dimer defect is placed in the lattice. Now we must elucidate what happens close to this resonant energy when several dimer defects are placed at random in the lattice, in comparison with lattices with the same number of unpaired defects. We used lattice sizes ranging from $N=2000$ to $N=500000$ sites. The largest of these systems are physically unrealizable, but it is important to study theoretically those systems to clearly elucidate the truly extended character of states close to the resonant energy, as we will demonstrate in the rest of the paper; the results for the smallest values are those directly related to experiments. Concerning the fraction of impurities, we only present here values corresponding to low defect concentration ( $c$ ranging from 0.1 to 0.3 ) because of their more physical relevance to actual systems, but we should stress that the main conclusion of the paper, namely, the existence of truly extended states in quasi-one-dimensional lattices with correlated disorder, is independent of $c$.

\section{A. Transmission coefficient}

Since we are dealing with random lattices, we will need ensemble averages to compute the transmission coefficient. Some years ago, Sak and $\mathrm{Kramer}^{38}$ pointed out that only its logarithm obeys the central limit theorem, thus being the unique physically representative magnitude of the electron transmission, rather than the transmission coefficient itself or its inverse. Therefore we have actually computed $\exp \langle\ln \tau(E)\rangle$, where $\langle\cdots\rangle$ means ensemble average. Nevertheless, in what follows we refer to this quantity simply as the transmission coefficient and denote it by $\tau$, but it is understood that averages are carried out over the logarithm.

An example of the behavior of $\tau$ around the resonant energy is shown in Fig. 1, for both paired as well as unpaired lattices, with the same values of $c=0.1$ and size $N=2000$. A careful inspection of the figure clearly reveals that $\tau$ is at least two orders of magnitude larger in paired lattices than in unpaired ones in the region of interest. In addition, and what is more apparent, $\tau$ is close to unity around the resonant energy $-4.73 \mathrm{eV}$, hence indicating that perfect transparency is preserved even when a finite number of dimer defects is placed at random in the lattice. This is a signature of the existence of a band of extended states close to that energy. We stress that, in spite of the fact that the plot corresponds to an average of over 300 realizations, the transmission coefficient for typical realizations always behaves in the same manner, 


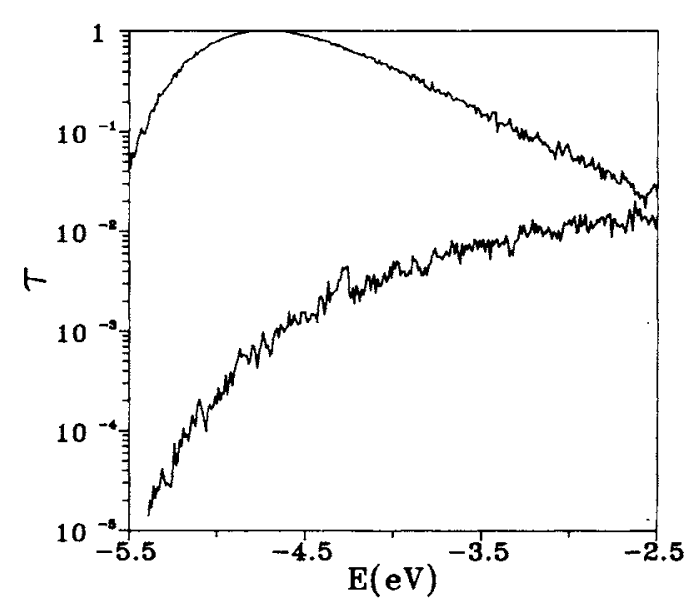

FIG. 1. Transmission coefficient as a function of the energy around the resonant energy $-4.73 \mathrm{eV}$, for paired (upper curve) and unpaired (lower curve) random lattices. Shown are averages over 300 realizations. Every realization consists of $N=2000$ scatterers and a fraction of defects, $c=0.1$.

although the plots are noisier. Thus, the only appreciable effect of averaging is to smooth out some particular very narrow, realization-dependent peaks, keeping the main common wide peak centered at the resonant energy.

We want to highlight that the width of the transmission peak is always nonzero. Hence, close to the resonant energy, there is an interval of energies that also shows high transparency, similar to that of the resonant energy (note that there is a difference of about three orders of magnitude between the transmission coefficient of paired and unpaired lattices in that interval). The peak width depends on the concentration of dimers: The larger the concentration, the narrower the peak, being always of finite width as already stated. Figure 2 shows results for

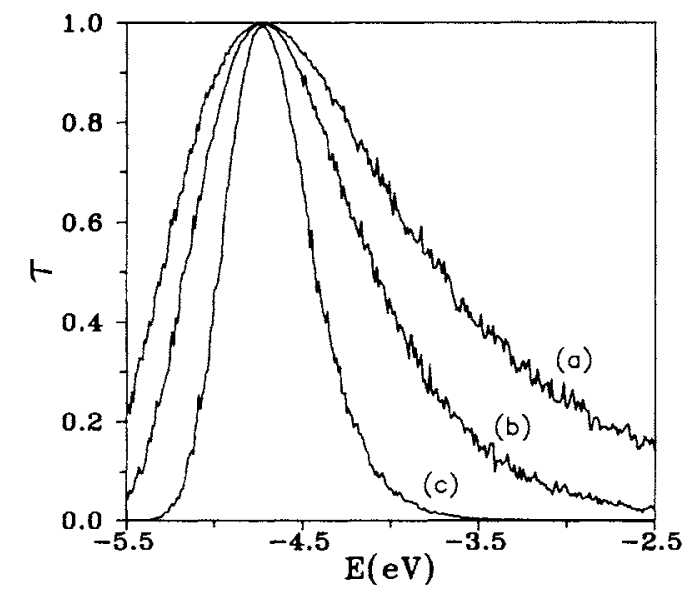

FIG. 2. Transmission coefficient as a function of the energy around the resonant energy $-4.73 \mathrm{eV}$ for paired lattices with (a) $N=1000$ and $c=0.1$, (b) $N=2000$ and $c=0.1$, and (c) $N=2000$ and $c=0.3$. Shown are averages over 300 realizations. two different values of $c(0.1$ and 0.3$)$ and $N=2000$. In addition, the width depends also on the system size: The larger the size, the narrower the peak, as shown in Fig. 2 for two values of $N(1000$ and 2000) for $c=0.1$. It is worth mentioning that $\tau$ is always unity at the resonant energy, irrespective of the value of $c$ or $N$.

\section{B. Lyapunov coefficient}

The fact that around the resonant energy $\tau$ approaches unity suggests the possibility that the localization length of those states may be very large. This is, in fact, what is deduced from the analysis of the Lyapunov coefficient (recall that it is the inverse of the localization length). Results are plotted in Fig. 3 for paired as well as unpaired random lattices with $N=2000$ and $c=0.1$. The comparison between the results for the two kinds of lattices is actually dramatic. First of all, we again observe that there exists a difference of several orders of magnitude between the localization length in both systems. In addition, paired lattices reflect the fact that a large number of states around the resonant energy presents a very large localization length (which manifests itself in a deep minimum of $\Gamma$, with the Lyapunov coefficient taking values of the order of the inverse of the system size in a nonzero width region), whereas there is a monotonic dependence of the Lyapunov coefficient for unpaired disorder.

\section{Multifractal analysis}

From the study of the transmission coefficient and the Lyapunov coefficient we are led to the conclusion that there exists a large number of electronic states that remains unscattered (or almost unscattered) by dimer defects. Such states are characterized by very large localization lengths (conversely, very small Lyapunov coeff-

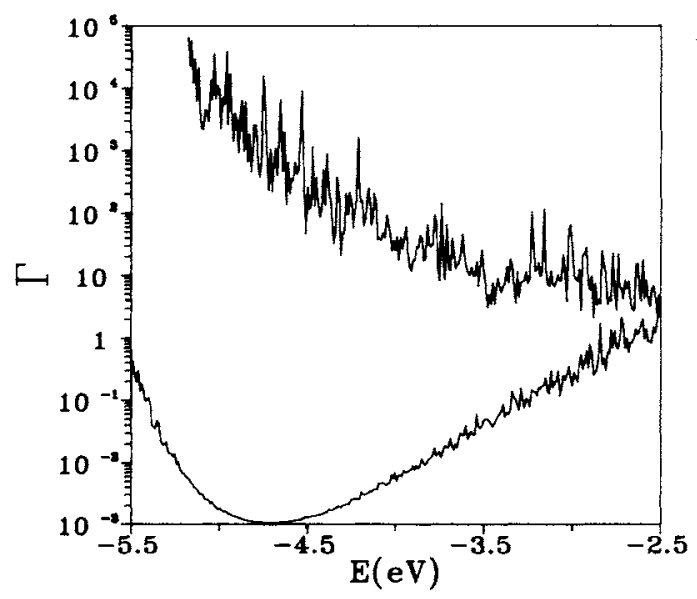

FIG. 3. Lyapunov coefficient as a function of the energy around the resonant energy $-4.73 \mathrm{eV}$, for paired (lower curve) and unpaired (upper curve) random lattices. Shown are averages over 300 realizations. Every realization consists of $N=2000$ scatterers and a fraction of defects, $c=0.1$. 
cients). Nevertheless, this result does not necessarily imply that those states are truly extended, namely, states that cannot be normalized in the thermodynamic limit. Then we must search for a different approach in order to elucidate the localized or extended character of the eigenstates. The characterization of the spatial extent of the wave function to all length scales may be accomplished by means of multifractal analysis. ${ }^{39}$ Extended states span homogeneously the whole lattice whereas localized states remain confined in finite regions. The amplitude distribution of the electronic states can be characterized by the scaling with the system size of moments associated to the measure defined in the system by us (in our case the probability of finding the electron at a given point):

$$
\mu_{q}(N)=\frac{\sum_{n=1}^{N}\left|\chi_{n}\right|^{2 q}}{\left(\sum_{n=1}^{N}\left|\chi_{n}\right|^{2}\right)^{q}}
$$

Notice that the second moment $\mu_{2}(N)$ coincides with the inverse participation ratio (IPR) as introduced, for instance, in Ref. 40. The generalized dimensions $D_{q}$ are determined via the scaling $\mu_{q}(N) \sim N^{-(q-1)} D_{q}$, for $q \neq 1$. For localized states $D_{q}$ vanishes for all $q$ whereas $D_{q}$ equals unity for states spreading uniformly. In previous works ${ }^{12,13}$ we have proved that multifractal analysis is a powerful tool to reveal the existence of truly extended states in $1 \mathrm{D}$ random systems (phonons, electrons) with correlated disorder. Hence, we expect that similar characterization techniques also work well in quasi-onedimensional systems, as in the present case.

Let us start with the IPR. From its definition, it can be seen that delocalized states are expected to present small values of the IPR, of order of $1 / N$, while localized states have much larger values. In the extreme case, when the electron is localized at a single site, Eq. (27) implies that $\mu_{q}(N)=1$. A typical situation is presented in Fig. 4 for the same system parameters as in Fig. 1, using the initial conditions $\chi_{0}=0$ and $\chi_{1}=1$ to iterate the equation of

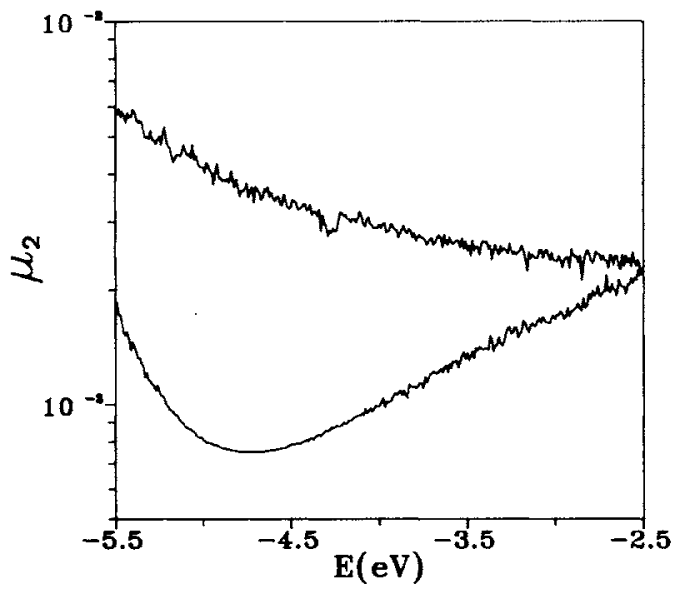

FIG. 4. IPR ( $\left.\mu_{2}\right)$ as a function of the energy around the resonant energy $-4.73 \mathrm{eV}$, for paired (lower curve) and unpaired (upper curve) random lattices, with the same parameters as in Fig. 1.

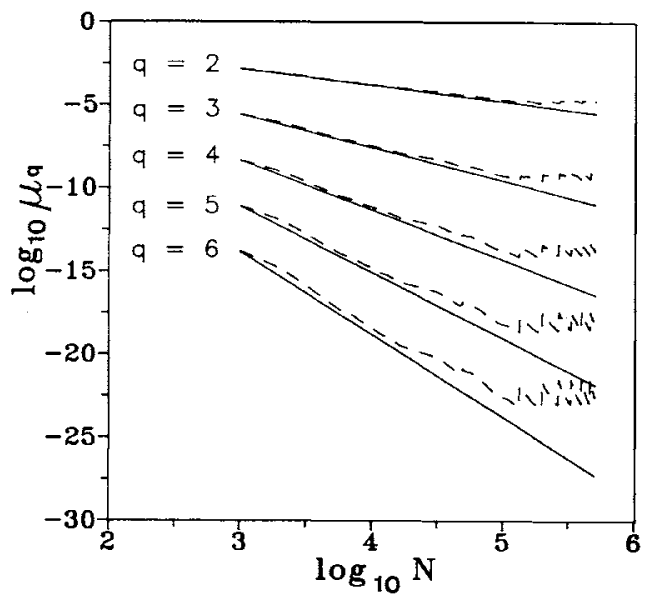

FIG. 5. Scaling of moments $\mu_{2}-\mu_{6}$ with the system size in paired random lattices for an energy $-4.70 \mathrm{eV}$ (solid lines), i.e., close to the resonant energy, and for an energy $-4.50 \mathrm{eV}$ (dashed lines), i.e., far from the resonant energy. Defect concentration is $c=0.1$.

motion given by Eq. (12) in order to find $\chi_{k}$. One can observe a wide, deep minimum of the IPR around the resonant energy for the paired disordered lattices, whereas this minimum is completely absent in the unpaired one. It is important to mention here that the value of the IPR at the minimum is independent of the defect concentration $c$, and depends only on the system size $N$. This suggests that the exact number of dimer defects is immaterial as far as the existence of such extended states is concerned.

A complete multifractal analysis requires one to study the scaling of all moments, defined by Eq. (27), with system size. We have considered such scaling for $q=$ $2,3, \ldots, 6$, and results are plotted in Fig. 5 for a concentration of $c=0.1$ of dimer defects. We have observed that those moments scale very accurately as $\mu_{q}(N) \sim N^{-(q-1)}$ for energies close to the resonant one, as illustrated in Fig. 5 for $-4.70 \mathrm{eV}$ (close to but not the exact resonant energy). On the contrary, for more distant energies $\mu_{q}(N)$ follows a power law for small systems but tends to a constant value for larger ones, as seen in Fig. 5 for $-4.50 \mathrm{eV}$. Therefore, according to the above discussion, the generalized dimensions $D_{q}$ are exactly unity, within numerical accuracy, for states close to the resonant energy, thus indicating the truly extended character of such states, in agreement with results obtained from the analysis of the IPR above.

\section{CONCLUSIONS}

In this paper we have considered electron dynamics in quasi-one-dimensional models of polymers with correlated disorder, and we have compared our results to those obtained in systems with ordinary (uncorrelated) disorder. Our procedure based on the NLP allows us to carry out a fully three-dimensional analysis of the model, with 
the scatterers placed along a straight line. It is important to realize that this technique can be made exact, and so there are no theoretical limitations on this approach. In addition, the exact solution can be found for an arbitrary NLP, as we actually demonstrated [see Eq. (4)] by means of a Fourier transform (which, in fact, is completely equivalent to use a Green's function formalism). As our selection for a suitable NLP that can reproduce experimental data for polymers, we have used surface $\delta$ function interactions with vanishing radius since, as we have previously shown, ${ }^{26}$ this potential gives very accurate results in the context of polymers. Using this model, we have found that there exists a resonant energy for which the reflection coefficient of a single dimer defect vanishes; that is, there is perfect transparency. Afterwards, we turned to the problem of electron scattering when several of such defects are located at random in the lattice. Results from the evaluation of the transmission coefficient and Lyapunov coefficient (the inverse of the localization length) strongly suggest that there exist many states close to the resonant energy that remain unscattered, where this is not the case when the constraint of pairing is relaxed. To demonstrate that such states are actually extended in nature, we have used multifractal analysis, which confirms our claim.

We now stress the physical relevance of our results. A key observation is that the resonant energy value does not depends on the defect concentration $c$. Therefore, by modifying this concentration, we could shift the Fermi level of the quasi-one-dimensional lattice to match the resonance. In this case, when the Fermi level reaches the resonant energy, a large increase should be observed in the electrical conductance peak. In fact, we have recently demonstrated in 1D Kronig-Penney models with correlated disorder that very noticeable peaks in the finite- temperature dc conductance appear as the Fermi level is moved through the band of extended states. ${ }^{17}$ In a similar way, one could expect such a dramatic increase in more elaborated models, as is the case with the one we present here. On the other hand, our model supports results previously found for simpler, pure $1 \mathrm{D}$ ones. ${ }^{5}$ It seems to us that this agreement makes very appealing the idea of the use polymeric systems to confirm experimentally the existence of delocalized states in $1 \mathrm{D}$ models, for what we have shown is that $3 \mathrm{D}$ effects do not destroy the coherence required for those states to appear. In fact, it is very tempting to relate all this to the known fact that polyaniline shows a metal-insulator transition, with the concentration of dopant acting as a control parameter (see Ref. 41 and references therein). Another important consequence of this work is that other experimental procedures we have proposed to find out whether delocalization can be measured or not, such as disordered superlattices, ${ }^{17}$ are likely to be correct even if $3 \mathrm{D}$ effects have not been taken into account. We hope that this result encourages experimental work in the field of quasione-dimensional disordered systems which could give status of physically relevant to the theoretically predicted bands of delocalized states.

\section{ACKNOWLEDGMENTS}

We thank collaboration and illuminating conversations with E. Maciá. Work at Madrid is supported by UCM through Project No. PR161/93-4811. Work at Leganés is supported by the DGICyT (Spain) through Project No. PB92-0248, and by the European Union Human Capital and Mobility Programme through Contract No. ERBCHRXC'T930413.
${ }^{1}$ J. C. Flores, J. Phys. Condens. Matter 1, 8471 (1989).

${ }^{2}$ D. H. Dunlap, H.-L. Wu, and P. Phillips, Phys. Rev. Lett. 65, 88 (1990).

${ }^{3}$ H.-L. Wu and P. Phillips, J. Chem. Phys. 93, 7369 (1990).

${ }^{4}$ H.-L. Wu and P. Phillips, Phys. Rev. Lett. 66, 1366 (1991).

${ }^{5}$ P. Phillips and H.-L. Wu, Science 252, 1805 (1991).

${ }^{6}$ A. Bovier, J. Phys. A 25, 1021 (1992).

${ }^{7}$ H.-L. Wu, W. Goff, and P. Phillips, Phys. Rev. B 45, 1623 (1992).

${ }^{8}$ S. N. Evangelou and D. E. Katsanos, Phys. Lett. A 164, 456 (1992).

${ }^{9}$ P. K. Datta, D. Giri, and K. Kundu, Phys. Rev. B 47, 10727 (1993).

${ }^{10}$ S. N. Evangelou and A. Z. Wang, Phys. Rev. B 47, 13126 (1993).

11 J. C. Flores and M. Hilke, J. Phys. A 26, L1255 (1993).

${ }^{12}$ F. Domínguez-Adame, E. Maciá, and A. Sánchez, Phys. Rev. B 48, 6054 (1993).

${ }^{13}$ A. Sánchez, E. Maciá, and F. Domínguez-Adame, Phys. Rev. B 49, 147 (1994); 49, 15 428(E) (1994).

${ }^{14}$ A. Sánchez and F. Domínguez-Adame, J. Phys. A 27, 3725 (1994).
${ }^{15}$ S. N. Evangelou, A. Z. Wang, and S. J. Xiong, J. Phys. Condens. Matter 6, 4937 (1994).

${ }^{16}$ P. K. Datta and K. Kundu, J. Phys. Condens. Matter 6, 4465 (1994).

${ }^{17}$ E. Diez, A. Sánchez, and F. Domínguez-Adame, Phys. Rev. B 50, 14359 (1994).

${ }^{18}$ R. Brito, F. Domínguez-Adame, and A. Sánchez (unpublished).

${ }^{19}$ J. M. Ziman, Models of Disorder (Cambridge University Press, London, 1979).

${ }^{20}$ F. Domínguez-Adame, B. Méndez, A. Sánchez, and E. Maciá, Phys. Rev. B 49, 3839 (1994).

${ }^{21}$ F. Domínguez-Adame, E. Maciá, and A. Sánchez, Phys. Rev. B 50,6453 (1994).

${ }^{22}$ A. Sánchez, F. Domínguez-Adame, and E. Maciá, Phys. Rev. B 51, 173 (1995).

${ }^{23}$ M. D. Stephens and J. L. Skinner, Chem. Phys. 177, 727 (1993).

${ }^{24}$ B. W. Knight and G. A. Peterson, Phys. Rev. 132, 1085 (1963).

${ }^{25}$ P. R. Sievert and M. L. Glasser, Phys. Rev. B 7, 1265 (1973). 
${ }^{26}$ F. Domínguez-Adame, B. Méndez, E. Maciá, and M. A. González, Mol. Phys. 74, 1065 (1991).

${ }^{27}$ A. J. Heeger, S. Kivelson, J. R. Schrieffer, and W. P. Su, Rev. Mod. Phys. 60, 781 (1988).

${ }^{28}$ A. Sánchez and L. Vázquez, Int. J. Mod. Phys. B 5, 2825 (1991); S. A. Gredeskul and Yu. S. Kivshar, Phys. Rep. 216, 1 (1992).

${ }^{29}$ S. A. Gredeskul, Yu. S. Kivshar, L. K. Maslov, A. Sánchez, and L. Vázquez, Phys. Rev. A 45, 8867 (1992).

${ }^{30}$ R. L. Kronig and W. B. Penney, Proc. R. Soc. London A 130, 499 (1931).

${ }^{31}$ M. L. Glasser, Surf. Sci. 64, 141 (1977).

${ }^{32}$ Y. Yamaguchi, Phys. Rev. 95, 1628 (1954).

${ }^{33}$ A. Galindo and P. Pascual, Quantum Mechanics (Springer,
Berlin, 1990)

${ }^{34}$ P. D. Kirkman and J. B. Pendry, J. Phys. C 17, 4327 (1984).

${ }^{35}$ R. E. Borland, Proc. R. Soc. London A 274, 529 (1963).

${ }^{36}$ J. Fink and G. Leising, Phys. Rev. B 34, 5320 (1986).

${ }^{37}$ P. M. Grant and I. P. Batra, Solid State Commun. 29, 225 (1979).

${ }^{38}$ J. Sak and B. Kramer, Phys. Rev. B 24, 1761 (1981).

${ }^{39}$ M. Schreiber and H. Grussbach, Mod. Phys. Lett. B 6, 851 (1992).

${ }^{40} \mathrm{~J}$. Canisius and J. L. van Hemmen, J. Phys. C 18, 4873 (1985).

${ }^{41}$ H. Dücker, M. Struck, Th. Koslowski, and W. von Niessen, Phys. Rev. B 46, 13078 (1992). 